\title{
Fragilariaceae (Bacillariophyceae) de rios da bacia do Iguaçu, Estado do Paraná, Brasil ${ }^{1}$
}

\author{
NICOLE M. BRASSAC ${ }^{2,4}$ e THELMA A.V.LUDWIG ${ }^{3}$
}

(recebido: 25 de julho de 2002; aceito: 14 de maio de 2003)

\begin{abstract}
Fragilariaceae (Bacillariophyceae) from rivers of Iguaçu basin, Paraná State, Brazil). This is a survey of the diatom flora of rivers in the influenced area of Caxias' Power Plant, in the district of Capitão Leônidas Marques, southwest of Paraná State, Brazil. Samples were collected from March 1997 to February 1998, in eight sampling stations in the Iguaçu River and its tributaries: Cotegipe, Jaracatiá, Chopim, Tormenta, Adelaide, and Guarani rivers. Nine taxa belonging to the family Fragilariaceae were identified: Asterionella formosa Hassal var. formosa, Fragilaria capucina var. fragilarioides (Grunow) Ludwig \& Flôres, F. capucina var. vaucheriae (Kützing) Lange-Bertalot, F. delicatissima (Wm. Smith) Lange-Bertalot var. delicatissima, F. javanica Hustedt var. javanica, Martyana martyi (Héribaud) Round var. martyi, Pseudostaurosira brevistriata (Grunow) Williams \& Round var. brevistriata, Synedra goulardii Brébisson var. goulardii and S. ulna (Nitzsch) Ehrenberg var. ulna. Martyana martyi and Fragilaria delicatissima were reported for the first time for Paraná State.
\end{abstract}

Key words - diatoms, Fragilariaceae, Iguaçu river, lotic ecosystem, river basin

RESUMO - (Fragilariaceae (Bacillariophyceae) de rios da Bacia do Iguaçu, Estado do Paraná, Brasil). Este trabalho é uma contribuição ao inventário das diatomáceas dos rios da área a ser inundada para a construção do reservatório da Usina Hidrelétrica de Salto Caxias, localizada no Município de Capitão Leônidas Marques, sudoeste do Estado do Paraná, Brasil. Coletas foram realizadas, mensalmente, de março de 1997 a fevereiro de 1998, em 8 estações de amostragem, abrangendo o Rio Iguaçu e seus afluentes, os rios Cotegipe, Jaracatiá, Chopim, Tormenta, Adelaide e Guarani. Nove táxons infragenéricos pertencentes à família Fragilariaceae foram identificados: Asterionella formosa Hassal var. formosa, Fragilaria capucina var. fragilarioides (Grunow) Ludwig \& Flôres, F. capucina var. vaucheriae (Kützing) Lange-Bertalot, F. delicatissima (Wm. Smith) Lange-Bertalot var. delicatissima, F. javanica Hustedt var. javanica, Martyana martyi (Héribaud) Round var. martyi, Pseudostaurosira brevistriata (Grunow) Williams \& Round var. brevistriata, Synedra goulardii Brébisson var. goulardii e S. ulna (Nitzsch) Ehrenberg var. ulna. Martyana martyi e Fragilaria delicatissima foram primeiras citações para o Estado do Paraná.

Palavras-chave - bacia hidrográfica, diatomáceas, ecossistemas lóticos, Fragilariaceae, Rio Iguaçu

\section{Introdução}

A flora diatomológica de ecossistemas lóticos continentais tem sido pouco estudada considerando a extensão da rede hidrográfica brasileira. A maioria das publicações está concentrada nos Estados do Paraná e Rio Grande do Sul. Dissertações de mestrado reúnem grande parte das informações sobre a diatomoflora de ambientes lóticos paranaenses. Foram estudadas as bacias dos rios Iguaçu (Contin 1990, Brassac 1999), Itararé e Tibagi (Atab 2000), bacia Litorânea (Landucci 2002) e bacia metropolitana de Curitiba (Visinoni 2002). Córregos e outros rios foram estudados por Train

1. Parte de dissertação de Mestrado de N.M. Brassac.

2. Centro Universitário Positivo, Núcleo de Ciências Biológicas e da Saúde, Rua Professor Pedro Viriato Parigot de Souza, 5.300, 81280-330 Curitiba, PR, Brasil.

3. Universidade Federal do Paraná, Departamento de Botânica, Centro Politécnico, Caixa Postal 19031, 81531-970 Curitiba, PR, Brasil.

4. Autor para correspondência: nicole@unicenp.br
(1990), Moreira (1990) e Leandrini (1999). Pequenos inventários ou trabalhos sobre diatomáceas relacionadas à qualidade da água de rios e arroios do Rio Grande do Sul foram publicados (Côrte-Real \& Aguiar 1972, Martau et al. 1977, Torgan \& Aguiar 1978, LaudaresSilva 1987, Callegaro et al. 1993, Lobo et al. 1996). Para Santa Catarina, Rodrigues $(1984,1992)$ e Rodrigues \& Moreira Filho (1990) realizaram levantamentos sobre as diatomáceas do Rio Tubarão.

Alguns trabalhos resultaram de projetos de monitoramento do fitoplâncton em regiões a serem impactadas pelo represamento de rios, por ocasião da instalação de usinas hidrelétricas. Ludwig \& Flôres $(1995,1997)$ iniciaram o estudo da diatomoflórula de rios a serem represados para a geração de energia elétrica, na bacia do Rio Iguaçu, em que foram abordadas as classes Coscinodiscophyceae, Bacillariophyceae e Fragilariophyceae. Brassac et al. (1999) divulgaram o inventário das diatomáceas cêntricas dos rios da região de abrangência da Usina Hidrelétrica de Salto Caxias (Bacia do Iguaçu), apresentando 19 táxons 
infragenéricos. Bicudo et al. (1993) determinaram 51 táxons infragenéricos representantes de diatomáceas, sendo cinco da classe Fragilariaceae do Rio Paranapanema, trecho a represar para construção da Usina Hidrelétrica de Rosana em São Paulo.

Objetivou-se dar continuidade ao inventário florístico das diatomáceas de ecossistemas lóticos pertencentes à bacia hidrográfica do Iguaçu, avaliandose a problemática na identificação e a variabilidade morfológica, além das atualizações nomenclaturais ocorridas no grupo das Fragilariaceae estudadas.

\section{Material e métodos}

A Bacia Hidrográfica do Iguaçu é o maior complexo hídrico do Estado do Paraná, ocupando uma área de $55.024 \mathrm{~km}^{2}$ (Suderhsa 1995). A Usina Hidrelétrica de Salto Caxias, área de propriedade da Companhia Paranaense de Energia (Copel), opera no Rio Iguaçu, Município de Capitão Leônidas Marques, a sudoeste do Estado (Intertechne 1993). O estudo foi realizado em ambientes lóticos situados na área de influência do reservatório da usina, entre as coordenadas $25^{\circ} 23^{\prime}-25^{\circ} 40^{\prime}$ S e $53^{\circ} 02^{\prime}-53^{\circ} 30^{\prime} \mathrm{W}$, abrangendo cinco municípios paranaenses.

Selecionaram-se oito estações de coleta, localizadas no próprio Rio Iguaçu e afluentes, a saber: estação 1 - Rio Iguaçu, próximo ao eixo da barragem, Município de Capitão Leônidas Marques; estação 2 - Rio Cotegipe, Município de Nova Prata do Iguaçu; estação 3 - Rio Adelaide, Município de Três Barras do Paraná; estação 4 - Rio Tormenta, Município de Três Barras do Paraná; estação 5 - Rio Guarani, próximo à reserva do Guarani, Município de Três Barras do Paraná; estação 6 Rio Jaracatiá, Município de Salto do Lontra; estação 7 - Rio Chopim, Município de Barra do Chopim; estação 8 - Rio Iguaçu, Município de Barra do Chopim.

Coletas mensais foram realizadas durante 12 meses, de março/1997 a fevereiro/1998, através de rede de fitoplâncton (abertura de malha de $25 \mu \mathrm{m}$ ), e fixadas com solução aquosa de formol a 4\%. Amostras líquidas encontram-se tombadas no Herbário da Universidade Federal do Paraná (UPCB 34879 a 35047). Lâminas permanentes foram utilizadas para o estudo qualitativo das diatomáceas, uma série confeccionada com material não oxidado e outra com amostras submetidas à oxidação (Moreira Filho \& ValenteMoreira 1981), utilizando-se Nafrax como meio de inclusão. As ilustrações foram obtidas através de fotomicrografias com filmes Copex Rapid (Agfa) e Imagelink HQ (Kodak).

\section{Resultados e Discussão}

A família Fragilariaceae nos rios da área de abrangência da Usina Hidrelétrica de Caxias está representada por nove táxons infragenéricos, distribuídos em cinco gêneros, descritos a seguir.

\section{Asterionella Hassal}

Asterionella formosa Hassal var. formosa, Microscopic Examination of the Water p.10, pl.2, fig.5. 1855.

Figura 1.

Valvas lineares, heteropolares; extremidades capitadas; área axial linear, estreita, de difícil visualização; área central ausente; estrias paralelas. Eixo apical: 46,4-85,0 $\mu \mathrm{m}$; eixo transapical: 2,0-3,2 $\mu \mathrm{m}$; 18-20 estrias em $10 \mu \mathrm{m}$.

Distribuição no Estado do Paraná: Mangueirinha, Pinhão e Palmas: Ludwig \& Flôres (1995); Ponta Grossa: Moro et al. (1994); Moro \& Fürstenberger (1993).

Distribuição na área de estudo: estações 1 e 8 (UPCB 34987, 34990, 35002, 35005, 35017, 35020, 35032, 35035)

A população de Asterionella formosa apresentou menor número de estrias no intervalo de $10 \mu \mathrm{m}$ do que o citado por Patrick \& Reimer (1966) de 24 a 28 em $10 \mu \mathrm{m}$, ampliando-se o limite mínimo de estrias do táxon.

\section{Fragilaria Lyngbye}

Apesar do tipo do gênero Fragilaria basear-se em uma espécie marinha, Round et al. (1990) sugerem que este gênero seja utilizado apenas para espécies de água doce. Lange-Bertalot (1980) e Krammer \& LangeBertalot (1991) incluem espécies de Synedra no gênero Fragilaria, acreditando não se justificar a separação entre os gêneros. Entretanto, mesmo reconhecendo a dificuldade na distinção entre Fragilaria e Synedra, Round (1991) elege o padrão de estriação como uma característica importante para separar os gêneros. Fragilaria caracteriza-se pelas estrias alternadas (as de uma margem intercaladas com as da margem oposta) e Synedra apresenta estrias opostas umas às outras. Além desta característica, o mesmo autor ressalta outras três, visíveis apenas sob microscopia eletrônica de varredura: 1) presença de cópula fechada em Synedra e aberta em Fragilaria; 2) presença de rimopórtula em ambas as extremidades no gênero Synedra e apenas em uma extremidade em Fragilaria; 3) padrão de desenvolvimento de campos de poros apicais nos dois gêneros. O padrão de estriação, caráter visível sob microscopia óptica, foi utilizado aqui, como distintivo entre Synedra e Fragilaria, mas concorda-se que os critérios ultra-estruturais definidos por Round (1991) são suficientes para a manutenção dos gêneros. 
Chave para os táxons constatados

1. Área central ausente Fragilaria javanica var. javanica

1. Área central presente

2. Área central intumescida unilateralmente Fragilaria capucina var. vaucheriae

2. Área central intumescida bilateralmente

3. Eixo apical com 20,0-50,4 $\mu \mathrm{m}$ Fragilaria capucina var. fragilarioides

3. Eixo apical com de $61,6-226,0 \mu \mathrm{m}$ Fragilaria delicatissima var. delicatissima

Fragilaria capucina Dèsmazieres var. fragilarioides (Grunow) Ludwig \& Flôres, Hoehnea 24(1):55. 1997. Figuras 2-4.

Valvas linear-lanceoladas; extremidades capitadas a subcapitadas; área axial estreita; área central aproximadamente retangular, intumescida bilateralmente; estrias paralelas, posição intercalada em relação às estrias da margem oposta. Eixo apical: 20,0-50,4 $\mu \mathrm{m}$; eixo transapical: $2,4-7,0 \mu \mathrm{m} ; 10-17$ estrias em $10 \mu \mathrm{m}$.

Distribuição no Estado do Paraná: Mangueirinha, Pinhão e Palmas: Ludwig \& Flôres (1997).

Distribuição na área de estudo: estações $1,2,3,4$, 5, 6 e 7 (UPCB 34879, 34882, 34884, 34889, 34890, 34891, 34911, 34904, 34905, 34906, 34918, 34922, $34924,34931,34932$, 34935, 34937, 34944, 34947, 34952, 34957, 34958, 34959, 34960, 34961, 34963, 34965, 34971, 34972, 34975, 34980, 34986, 34987, $34990,34995,34998,35001,35008,35010,35013$, $35016,35021,35023,35028,35031,35032$, 35035, 35036, 35038, 35040, 35046).

Ludwig \& Flôres (1997) comentam sobre a dificuldade na circunscrição das variedades de F. capucina. Concorda-se com esses autores, que caracterizam a variedade fragilarioides pela presença de menor número de estrias em $10 \mu \mathrm{m}$ (9-14), mostrando um padrão de estriação mais grosseiro e pela área central distintamente intumescida bilateralmente.

Fragilaria capucina Dèsmazieres var. vaucheriae (Kützing) Lange-Bertalot, Hedwigia 33:747, p1.1, fig.26-38, pl.4, fig.82-94, 97-102, pl.11, fig.216, 224, pl.12, fig.225, 233. 1980.

Figuras 5-8.

Valvas lanceoladas a linear-lanceoladas; extremidades capitadas, subcapitadas a rostradas; área axial estreitamente lanceolada a linear; área central aproximadamente retangular, com margem levemente intumescida em um dos lados; estrias paralelas, posição intercalada em relação às estrias da margem oposta.
Eixo apical: 7,2-28,0 $\mu \mathrm{m}$; eixo transapical: 2,0-5,6 $\mu \mathrm{m}$; 11-24 estrias em $10 \mu \mathrm{m}$.

Distribuição no Estado do Paraná: Mangueirinha, Pinhão e Palmas: Ludwig \& Flôres (1997).

Distribuição na área de estudo: estações $1,2,3$, 4, 5, 6, 7 e 8 (UPCB 34879, 34882, 34891, 34893, 34898, 34903, 34905, 34909, 34918, 349222, 34931, 34932, 34940, 34943, 34958, 34959, 34971, 34972, 34975, 34978, 34986, 34987, 34990, 35016, 35031, 35036).

De acordo com Patrick \& Reimer (1966), Fragilaria vaucheriae (Kützing) Boyer-Petersen apresenta área central assimétrica, em geral, intumescida unilateralmente. Adotou-se este critério diagnóstico para as determinações realizadas no presente trabalho. Os exemplares com extremidades capitadas poderiam ser identificados como Fragilaria vaucheriae (Kützing) Boyer-Petersen var. capitellata (Grunow) Ross. No entanto, Hustedt (1927-1930) ilustrou exemplares com extremidades sub-rostradas e Collins \& Kalinsky (1977), com valvas mais lineares e extremidades subcapitadas. Le Cohu \& Maillard (1986), em um estudo das diatomáceas de Kerguelen (França), encontraram uma população de Fragilaria capucina var. vaucheriae com grande variação morfológica, apresentando indivíduos de transição entre a forma vaucheriae e a forma capitellata.

A população encontrada na área de estudo mostrouse polimórfica em relação ao contorno valvar e à forma de extremidades, porém para o caráter diagnóstico (presença de área central intumescida unilaterialmente) foi constante.

Fragilaria delicatissima (Wm. Smith) Lange-Bertalot var. delicatissima, Nova Hedwigia 33:723. 1980. Figuras 9-11.

Valvas linear-lanceoladas; extremidades capitadas; área axial linear, estreita; área central retangular, levemente intumescida; estrias paralelas, posição intercalada com as da margem oposta. Eixo apical: 
61,6-226,0 $\mu \mathrm{m}$; eixo transapical: 2,0-5,0 $\mu \mathrm{m} ; 10-19$ estrias em $10 \mu \mathrm{m}$

Distribuição no Estado do Paraná: primeira citação do táxon.

Distribuição na área de estudo: estações 1,2 e 8 (UPCB 34879, 34880, 34906, 34918, 34932, 34944).

A população encontrada foi identificada dentro do gênero Fragilaria por apresentar estrias intercalares (ver comentários de Fragilaria capucina var. fragilarioides). Este táxon faz parte de um grupo taxonômico confuso, que envolve sinonimizações de espécies identificadas em Fragilaria e Synedra, mais especificamente variedades de Synedra acus Kützing (sensu Hustedt 1930) e Fragilaria delicatissima (Wm. Smith) Lange-Bertalot. A determinação dos indivíduos estudados baseou-se nas características diagnósticas apresentadas por Patrick \& Reimer (1966), pelas semelhanças métricas e morfológicas.

Fragilaria javanica Hustedt var. javanica, Archiv für Hydrobiologie 6(1):154, pl.10, fig.59-60. 1938.

Figura 12.

Valvas lineares, margens levemente constritas na porção mediana; extremidades subcapidatas a capitadas; áreas axial e central ausentes; estrias paralelas ao longo da superfície valvar. Eixo apical: 38,0-60,8 $\mu \mathrm{m}$; eixo transapical: 5,0-6,5 $\mu \mathrm{m} ; 21-23$ estrias em $10 \mu \mathrm{m}$.

Distribuição no Estado do Paraná: citada como Fragilaria constricta Ehrenberg var. constricta: Curitiba - Contin (1990).

Distribuição na área de estudo: estação 8 (UPCB 34987).

Fragilaria javanica é morfologicamente semelhante a Fragilariforma virescens (Ralfs) Williams \& Round. A distinção entre ambas baseia-se na formação de cadeias em zig-zag na primeira e lineares na segunda (Williams \& Round 1988). Além disso, em $F$. virescens observa-se área axial estreita, margens laterais paralelas e extremidades rostradas.

\section{Martyana Round}

Martyana martyi (Héribaud) Round var. martyi in Round, Crawford \& Mann, The Diatoms p.673. 1990. Figura 13.

Valvas lanceoladas; ápice arredondado, base atenuado-arredondada; área axial estreitamente lanceolada; estrias robustas, paralelas na porção mediana e radiadas nas extremidades. Eixo apical: $37,0 \mu \mathrm{m}$; eixo transapical: $6,0 \mu \mathrm{m}$; 6 estrias em $10 \mu \mathrm{m}$.
Distribuição no Estado do Paraná: primeira citação do táxon.

Distribuição na área de estudo: estação 8 (UPCB 34987).

De acordo com Round et al. (1990), o gênero Martyana está baseado em Opephora martyi, uma espécie dulceaqüícola que difere estruturalmente do gênero Opephora, originalmente marinho. Segundo esses autores, as formas marinhas apresentam uma rimopórtula na extremidade, diferente de Martyana, que se distingue também em outros aspectos, como estrutura das aréolas e ausência de espinhos marginais.

Pseudostaurosira (Grunow) Williams \& Round

Pseudostaurosira brevistriata (Grunow) Williams \& Round var. brevistriata, Diatom Research 2:276. 1987. Figura 14.

Valvas lanceoladas; extremidades rostradas; área axial e central formando área hialina amplamente lanceolada; estrias curtas e paralelas a levemente radiadas. Eixo axial: 24,8 $\mu \mathrm{m}$; eixo transapical: 7,2 $\mu \mathrm{m}$; 12 estrias em $10 \mu \mathrm{m}$.

Distribuição no Estado do Paraná: citado como Fragilaria brevistriata Grunow: Curitiba - Contin (1990), Lozovei \& Shirata (1990).

Distribuição na área de estudo: estação 4 (UPCB 34924).

Anteriormente pertencentes ao gênero Fragilaria, as espécies do gênero Pseudostaurosira são tipicamente dulceaqüícolas, apresentando valvas lineares a elípticas e aréolas marginais esparsas (Round et al. 1990). Segundo Patrick \& Reimer (1966), Fragilaria brevistriata Grunow (= Pseudostaurosira brevistriata) pode ser diferenciada de outras espécies do gênero Fragilaria por apresentar estrias marginais delicadas e encurtadas.

Synedra Ehrenberg

As espécies pertencentes a este gênero são tipicamente dulceaqüícolas, planctônicas ou bentônicas. A taxonomia deste grupo é bastante confusa, apresentando similaridades com Fragilaria, sendo que alguns autores (Lange-Bertalot 1980, Krammer \& Lange-Bertalot 1991) defendem a união destes dois gêneros em um. Várias de suas espécies, principalmente as marinhas, foram recentemente transferidas para outros gêneros como Catacombas, Ctenophora, Hyalosynedra, Neosynedra e Tabularia (Round et al. 1990). 
Chave para os táxons constatados

1. Valvas com constrição mediana Synedra goulardii var. goulardii

1. Valvas sem constrição mediana Synedra ulna var. ulna

Synedra goulardii Brébisson var. goulardii, in Cleve \& Grunow, Kongliga Svenska Vetenskaps-Akademiens Handlingar 17(2):107, pl.6, fig.119. 1880.

Figuras 15-17.

Valvas linear-lanceoladas a lanceoladas, com constrição mediana; extremidades atenuadoarredondadas, subcapitadas a capitadas; área axial linear, estreita; área central quadrangular; estrias paralelas, alinhadas com as da margem oposta. Eixo apical: 56,8-128,0 $\mu \mathrm{m}$; eixo transapical: 7,2-12,0 $\mu \mathrm{m}$; 9-16 estrias em $10 \mu \mathrm{m}$.

Distribuição no Estado do Paraná: Curitiba: Contin (1990), Lozovei \& Shirata (1990); Mangueirinha, Pinhão e Palmas: Ludwig \& Flôres (1997).

Distribuição na área de estudo: estações $1,2,3,4$, 5, 6, 7 e 8 (UPCB 34879, 34880, 34882, 34884, 34887, 34889, 34890, 34891, 34893, 34894, 34896, 34898, 34901, 34903, 34904, 34905, 34906, 34909, 34911, 34914, 34916, 34918, 34919, 34920, 34922, 34924, $34927,34930,34931,34932$, 34940, 34942, 34943, $34947,34948,34950,34952$, 34955, 34957, 34958, 34959 , 34963, 34971, 34972, 34975, 34986, 34998, $35001,35005,35016,35017,35020,35021,35023$, $35025,35028,35031,35032,35035,35036,35038$, $35043,35046)$.

A população estudada mostrou-se bastante polimórfica, apresentando constrição valvar mediana sutil até fortemente pronunciada. Os exemplares da bacia do Rio Iguaçu apresentaram valores métricos mais amplos (tanto do eixo apical, quanto do transapical), assim como constatado por Rodrigues (1984) para material do Rio Tubarão. Entretanto, morfologicamente, são idênticos aos materiais apresentados por Patrick \& Reimer (1966) e Krammer \& Lange-Bertalot (1986).

Synedra ulna (Nitzsch) Ehrenberg var. ulna, Verh. Kgl. Akad. Wiss. Berlin p.53. 1836.

Figuras 18-19.

Valvas lineares a linear-lanceoladas, sem constrição mediana; extremidades rostradas a subcapitadas; área axial linear; área central quadrangular; estrias paralelas, alinhadas com as da margem oposta. Eixo apical: 72,0-400,0 $\mu \mathrm{m}$; eixo transapical: $6,0-10,4 \mu \mathrm{m} ; 7-11$ estrias em $10 \mu \mathrm{m}$.
Distribuição no Estado do Paraná: Curitiba: Moreira Filho et al. (1973), Cecy et al. (1976), Contin (1990), Lozovei \& Shirata (1990); Mangueirinha, Pinhão e Palmas: Ludwig \& Flôres (1997); Porto Rico: Oliveira et al. (1994).

Distribuição na área de estudo: estações 1, 2, 3, 4, 5, 6, 7 e 8 (UPCB 34879, 34880, 34882, 34884, 34887, 34889, 34890, 34891, 34893, 34894, 34896, 34898, 34901, 34903, 34905, 34906, 34907, 34909, 34911, 34914, 34916, 34918, 34919, 34920, 34922, 34924, 34927, 34929, 34931, 34932, 34933, 34935, 34937, 34940, 34942, 34943, 34944, 34947, 34948, 34950, 34952, 34955, 34957, 34958, 34959, 34960, 34961, 34963, 34965, 34971, 34972, 34975, 34976, 34978, 34980, 34983, 34985, 34986, 34987, 34990, 34991, 34993, 34995, 34998, 35001, 35002, 35005, 35006,35008 , 35010, 35013, 35015, 35016, 35017, $35020,35021,35023,35025,35028,35030,35031$, 35032 , 35035, 35036, 35038, 35040, 35043, 35045, 35046).

Grande polimorfismo relacionado com medidas e contorno valvar tem sido referido em $S$. ulna (Hustedt 1930, Patrick \& Reimer 1966, Germain 1981). Esta variação levou à descrição de muitas variedades não típicas para esta espécie. De acordo com Patrick \& Reimer (1966) e Germain (1981), a variedade amphirhynchus (Ehrenberg) Grunow diferencia-se da variedade $u$ lna por apresentar valvas mais longas (180-250 $\mu \mathrm{m})$ e ausência de área central. Já, a variedade danica (Kützing) van Heurck diferencia-se pela forma da valva e por apresentar área central transversal, não atingindo as margens (Hustedt 1930, Germain 1981). A variedade spathulifera (Grunow) van Heurck também diferencia-se da variedade típica por apresentar extremidades espatuladas e área central não atingindo as margens (Hustedt 1930). Dentro da população estudada foram encontrados apenas exemplares que se enquadram dentro da circunscrição da variedade típica. No entanto, há necessidade de uma revisão desta espécie a fim de verificar se as variedades citadas acima são realmente táxons diferentes ou se apenas representam um contínuo de variações morfológicas da variedade típica. 

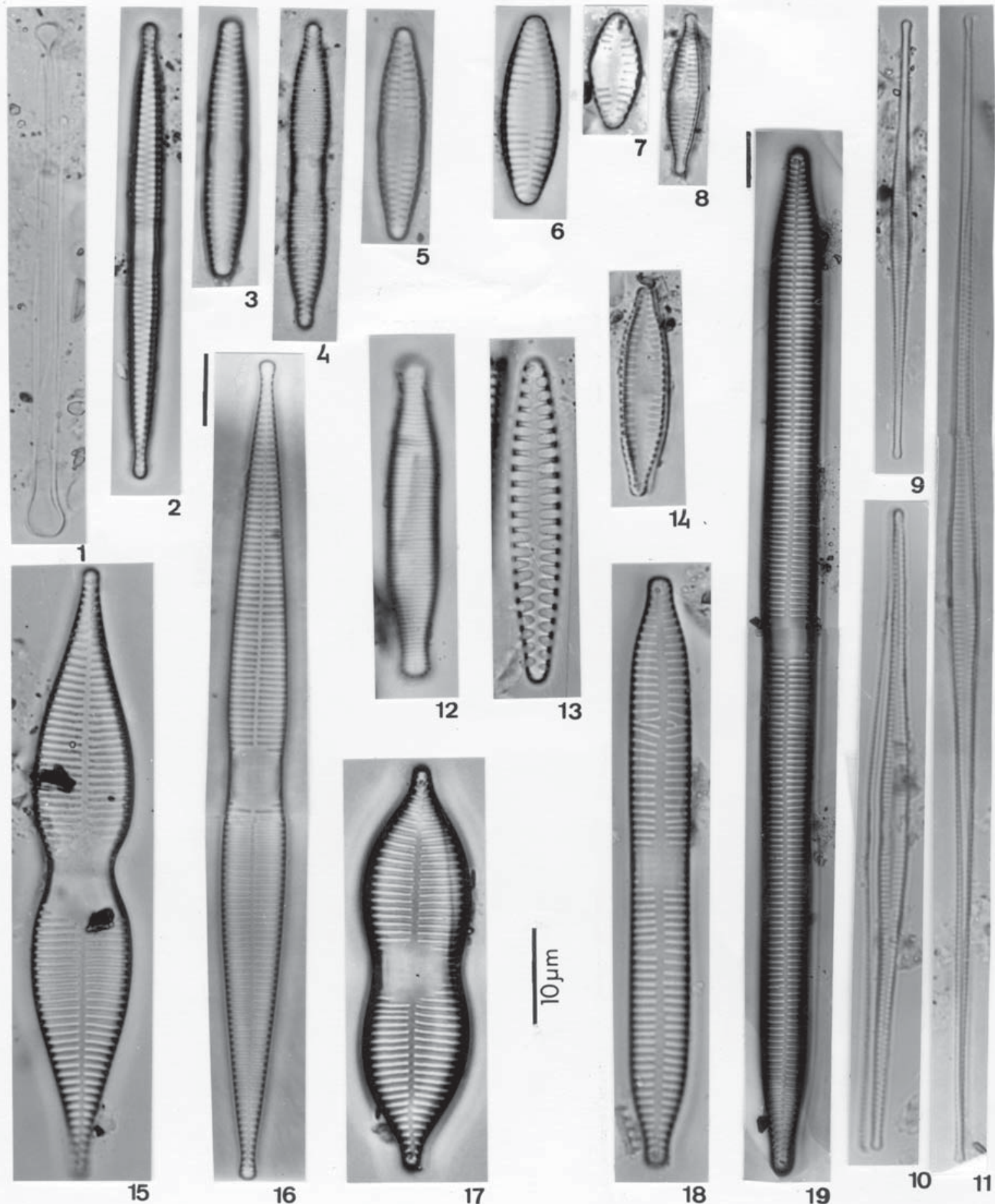

16

17

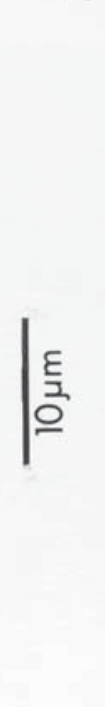

Figura 1. Asterionella formosa var. formosa. Figuras 2-4. Fragilaria capucina var. fragilarioides. Figuras 5-8. Fragilaria capucina var. vaucheriae. Figuras 9-11. Fragilaria delicatissima var. delicatissima. Figura 12. Fragilaria javanica var. javanica. Figura 13. Martyana martyi var. martyi. Figura 14. Pseudostaurosira brevistriata var. brevistriata. Figuras 15-17. Synedra goulardii var. goulardii. Figuras 18-19. Synedra ulna var. ulna. A escala inferior é geral, salvo quando especificamente indicado. Escalas $=10 \mu \mathrm{m}$.

Figure 1. Asterionella formosa var. formosa. Figures 2-4. Fragilaria capucina var. fragilarioides. Figures 5-8. Fragilaria capucina var. vaucheriae. Figures 9-11. Fragilaria delicatissima var. delicatissima. Figure 12. Fragilaria javanica var. javanica. Figure 13. Martyana martyi var. martyi. Figure 14. Pseudostaurosira brevistriata var. brevistriata. Figures 15-17. Synedra goulardii var. goulardii. Figures 18-19. Synedra ulna var. ulna. The scale below is general, except when specified. Scales = $10 \mu \mathrm{m}$. 
Agradecimentos - À equipe de campo da Companhia Paranaense de Energia - Copel, pelas coletas das amostras; ao Departamento de Genética BL/UFPR, pelo uso da sala de preparação fotográfica; ao $\mathrm{CNPq}$, pela concessão de bolsa de mestrado à primeira autora.

\section{Referências bibliográficas}

ATAB, D.R.R. 2000. Diatomáceas (Bacillariophyta) de rios das bacias do Tibagi e do Itararé, Centro-leste do Estado do Paraná. Dissertação de mestrado, Universidade Federal do Paraná, Curitiba.

BICUDO, D.C., BICUDO, C.E.M., CASTRO, A.M.J. \& PICELLI-VICENTIM, M.M. 1993. Diatomáceas (Bacillariophyceae) do trecho a represar do Rio Paranapanema (Usina Hidrelétrica de Rosana), Estado de São Paulo, Brasil. Hoehnea 20:47-68.

BRASSAC, N.M. 1999. Diatomoflórula dos rios da área de influência da Usina Hidrelétrica de Salto Caxias, bacia do Rio Iguaçu, Paraná. Dissertação de mestrado, Universidade Federal do Paraná, Curitiba.

BRASSAC, N.M., ATAB, D.R., LANDUCCI, M., VISINONI, N.D. \& LUDWIG, T.A.V. 1999. Diatomáceas cêntricas de rios na região de abrangência da Usina Hidrelétrica de Salto Caxias, PR (Bacia do Rio Iguaçu). Acta Botanica Brasilica 13:277-289.

CALLEGARO, V.L.M., SILVA, K.R.L. \& SALAMONI, S.E. 1993. Flórula diatomológica de ambientes lênticos e lóticos do Parque Florestal Estadual do Turvo, Rio Grande do Sul, Brasil. Iheringia, Série Botânica 43:89-134.

CECY, I.T., VALENTE-MOREIRA, I.M. \& HOHMANN, E. 1976. Estudos ficológicos e químico-bacteriológico da água do tanque do Passeio Público de Curitiba, Estado do Paraná, Brasil. Boletim do Museu Botânico Municipal 25:1-37.

COLLINS, B. \& KALLINSKY, R. 1977. Studies on Ohio diatoms. Bulletin of the Ohio Biological Survey 5:1-74.

CONTIN, L.F. 1990. Contribuição ao estudo das diatomáceas (Chrysophyta, Bacillariophyceae) na região de captação de água do Rio Iguaçu (SANEPAR), em Curitiba, Estado do Paraná, Brasil. Estudos de Biologia 24:1-95.

CÔRTE-REAL, M. \& AGUIAR, L.M. 1972. Diatomáceas do Arroio Dilúvio com referência às espécies de interesse sanitário e poluição. Iheringia, Série Botânica $16: 15-54$.

GERMAIN, H. 1981. Flore des Diatomées. Société Nouvelle des Éditions Boubée, Paris.

HUSTEDT, F. 1927-1930. Die kieselalgen. In KryptogamenFlora (L. Rabenhorst, ed.). Akademische Verlagsgesellschaf, Leipzig, v.7, n.1, p.1-920.

HUSTEDT, F. 1930. Bacillariophyta (Diatomeas). In Die Süsswasser-Flora Mitteleuropas (A. Pascher, ed.). G. Fischer, Jena, v.10, p.1-466.
INTERTECHNE. 1993. U.H. Salto Caxias, Rio Iguaçu, PR: Relatório de Impacto Ambiental. Companhia Paranaense de Energia - Copel, Curitiba.

KRAMMER, K. \& LANGE-BERTALOT, H. 1986. Bacillariophyceae: Naviculaceae. In Süsswasser Flora von Mittleleuropa (H. Ettl, I. Gerloff, H. Heynig \& D. Mollenhauer, eds.). G. Fischer, Jena, v.2, n.1, p.1-876.

KRAMMER, K. \& LANGE-BERTALOT, H. 1991. Bacillariophyceae: Centrales, Fragilariaceae, Eunotiaceae. In Süsswasser Flora von Mittleleuropa (H. Ettl, I. Gerloff, H. Heynig \& D. Mollenhauer, eds.). G. Fischer, Jena, v.2, n.3, p.1-576.

LANDUCCI, M. 2002. Diatomáceas de rios da Bacia Hidrográfica Litorânea, Paraná, Brasil: Coscinodiscophyceae, Fragilariophyceae e Bacillariophyceae (Achnanthales e Eunotiales). Dissertação de mestrado, Universidade Federal do Paraná, Curitiba.

LANGE-BERTALOT, H. 1980. Zur systematischen Bewertung der bandförmigen Kolonien von Navicula und Fragilaria. Kriterien für die Vereinigung von Synedra (subgen. Synedra) Ehrenberg mit Fragilaria Lyngbye. Nova Hedwigia 33:723-787.

LAUDARES-SILVA, R. 1987. Estudo taxonômico das diatomáceas (Bacillariophyceae) coletadas no Arroio do Faxinal (Sanga de Água Boa), Torres, Rio Grande do Sul. Insula 17:3-184.

LEANDRINI, J.A. 1999. Diatomáceas perifíticas da região de captação de água da SANEPAR, Maringá, PR (Córrego Sarandi e Rio Pirapó). Dissertação de mestrado, Universidade Federal do Paraná, Curitiba.

LE COHU, R. \& MAILLARD, R. 1986. Diatomeés d'eau douce de îles Kerguelen (à 1'exclusion de Monoraphideés). Annales Limnologie 22:99-118.

LOBO, E.A., CALLEGARO, V.L.M. \& OLIVEIRA, M.A. 1996. Pollution tolerant diatom from lotic systems in the Jacuí Basin, Rio Grande do Sul, Brasil. Iheringia, Série Botânica 47:45-72.

LOZOVEI, A.L. \& SHIRATA, M.T. 1990. Diatomáceas (Chrysophyta, Bacillariophyceae) no Rio Passaúna, Curitiba, Paraná, Brasil - Levantamento qualitativo da diatomoflórula em segmento manancial. Estudos de Biologia 27:5-56.

LUDWIG, T.A.V. \& FLÔRES, T. 1995. Diatomoflórula dos rios da região a ser inundada para a construção da Usina Hidrelétrica de Segredo, Paraná: I. Coscinodiscaceae, Bacillariophyceae (Achnanthales e Eunotiales) e Fragilariophyceae (Meridion e Asterionella). Arquivos de Biologia e Tecnologia 38:631-650.

LUDWIG, T.A.V. \& FLÔRES, T. 1997. Diatomoflórula dos rios da região a ser inundada para a construção da Usina Hidrelétrica de Segredo, Paraná: Fragilariophyceae (Fragilaria e Synedra). Hoehnea 24:55-65.

MARTAU, L., AGUIAR, L. \& CALLEGARO, V.L. 1977. Diatomáceas do Rio dos Sinos, Rio Grande do Sul, Brasil. Iheringia, Série Botânica 22:45-83. 
MOREIRA, A.L.O.R. 1990. Estudo taxonômico de Cymbella Agardh e Gomphonema Ehrenberg da região de captação de água do Rio Pirapó, Maringá, Paraná, Brasil. Dissertação de mestrado, Universidade Federal do Paraná, Curitiba.

MOREIRA FILHO, H. \& VALENTE-MOREIRA, I.M. 1981. Avaliação taxonômica e ecológica das diatomáceas (Bacillariophyceae) epífitas em algas pluricelulares obtidas nos litorais dos Estados do Paraná, Santa Catarina e São Paulo. Boletim do Museu Botânico Municipal 47:1-17.

MOREIRA FILHO, H., VALENTE-MOREIRA, I.M. \& CECY, I.T. 1973. Diatomáceas na barragem de captação d'água (Sanepar) do Rio Iguaçu, em Curitiba, Estado do Paraná. Acta Biologica Paranaense 2:133-145.

MORO, R.S. \& FÜRSTENBERGER, C.B. 1993. Diatomáceas (Bacillariophyceae) da Lagoa Dourada (Parque Estadual de Vila Velha), Paraná, Brasil. Acta Biologica Paranaense 22:15-30.

MORO, R.S., GARCIA, E. \& OLIVEIRA JÚNIOR, H.F. 1994. Diatomáceas (Bacillariohyceae) da Represa Alagados, Ponta Grossa, Brasil (exclusive cêntricas). Iheringia, Série Botânica 45:5-19.

OLIVEIRA, M.D., TRAIN, S. \& RODRIGUES, L.C. 1994. Levantamento preliminar do fitoplâncton de rede (exceto Zygnemaphyceae) do Rio Paraná, no Município de Porto Rico, Paraná, Brasil. Revista Unimar 16:155-174.

PATRICK, R. \& REIMER, C.W. 1966. The diatoms of United States. Academy of Natural Sciences, Philadelphia.

RODRIGUES, L. 1984. Contribuição ao conhecimento das diatomáceas do Rio Tubarão, Santa Catarina, Brasil. Insula 14:47-120.
RODRIGUES, L. 1992. Diatomoflórula do Rio Tubarão, Estado de Santa Catarina, Brasil: 2. Naviculaceae Kützing. Insula 21:3-41.

RODRIGUES, L. \& MOREIRA FILHO, H. 1990. Diatomoflórula do Rio Tubarão, Santa Catarina, Brasil: I - Eunotiaceae Kützing e Achnanthaceae Kützing. Insula 20:113-135.

ROUND, F.E. 1991. On striae patterns in Fragilaria and Synedra. Diatom Research 6:147-154.

ROUND, F.E., CRAWFORD, R.M. \& MANN, D.G. 1990. The diatoms - biology and morphology of the genera. Cambridge University Press, Cambridge.

SUDERHSA. 1995. Qualidade das Águas Interiores do Estado do Paraná: 1987-1995. Superintendência de Desenvolvimento de Recursos Hídricos e Saneamento Ambiental. Curitiba.

TRAIN, S. 1990. Diatomoflórula do Córrego Moscados, Município de Maringá, Estado do Paraná, Brasil. Dissertação de mestrado, Universidade Federal do Paraná, Curitiba.

TORGAN, L.C. \& AGUIAR, L. 1978. Diatomáceas do "Rio Guaíba", Porto Alegre, Brasil. Iheringia, Série Botânica 23:19-63.

VISINONI, N.C.D. 2002. Diatomáceas de rios da região metropolitana de Curitiba, Paraná, Brasil: Coscinodiscophyceae, Fragilariophyceae e Bacillariophyceae (Achnanthales e Bacillariales). Dissertação de mestrado, Universidade Federal do Paraná, Curitiba.

WILLIAMS, D.M. \& ROUND, F.E. 1988. Fragilariforma nom. nov., a new generic name for Neofragilaria Williams \& Round. Diatom Research 3:265-267. 